Proc. Indian Acad. Sci., Vol. 88 A, Part II, Number 1, March 1979, pp. 51-56, (c) printed in India.

\title{
Solar zenith angle and frequency dependence of ionospheric $D$-region absorption
}

\author{
S L JAIN* and R K RAI \\ Department of Physics, University of Udaipur, Udaipur 313001 \\ * Present address: Radio Science Division, National Physical Laboratory, \\ New Delhi 110012
}

MS received 1 April 1978; revised 16 December 1978

\begin{abstract}
In this paper, the results of a series of extensive measurements of multifrequency radio wave absorption in the $D$-region of the ionosphere during the epoch of the minimum period of the solar cycle are presented. Experimental results for Udaipur, a low latitude station $\left(24^{\circ} 35^{\prime} \mathrm{N}\right)$ are compared with the theoretically expected results. It is found that the experimental values of frequency index $m$ and $\cos \chi$ index $n$ agree well with those obtained theoretically. The mean values of $m$ and $n$ for the whole period of observation are found to be 1.76 and 1.4 respectively. The seasonal variation of the values of $m$ and $n$ is also discussed.
\end{abstract}

Keywords. Radiowave absorption; solar cycle.

\section{Introduction}

This paper reports the results of ionospheric absorption measurements of radiowaves at $2 \cdot 5,3 \cdot 0,3 \cdot 5$ and $4 \cdot 1 \mathrm{MHz}$. The measurements were made at Udaipur (Geog. Lat. $24^{\circ} 35^{\prime} \mathrm{N}$; Geog. Long. $73^{\circ} 42^{\prime}$ E) during the low solar activity period 1971-73. The equipment, the method of recording calibration and analysis of the data were reported earlier (Jain and Rai 1974 and 1976; Jain 1973).

\section{Analysis and results}

The non-deviative absorption of radio waves may be expressed in the form

$$
L \propto \frac{(\cos \chi)^{n}}{\left(f \pm\left|f_{L}\right|\right)^{m}}
$$

where the symbols have the usual meaning. The measured absorption represents the absorption in the region at and below the reflection points in the $E$ or $F$ layers. The $D$-region absorption is obtained by subtracting the absorption suffered in the $E$ and $F$ regions from the total absorption. The latter is calculated using Jaeger's (1947) method. 
At night there is an appreciable amount of absorption. This is attributed to ionisation due to cosmic rays as well as due to non-solar origin. This is accounted for by including a constant term in (1) as

$$
(L-A) \propto \frac{(\cos \chi)^{\infty}}{\left(\omega \pm\left|\omega_{\Sigma}\right|\right)^{m}}
$$

The value of $A$ is taken to be the average value of absorption at the working frequency. The $\cos \chi$ and frequency indices $n$ and $m$ have been graphically deter mined. The error in absorption values is estimated to be $10-15 \%$ in general and about $50 \%$ in some extreme cases.

Typical graphs and histograms for the indices $n$ and $m$ are shown in figures 1 to 4. Variation of the index $m$ with $\cos \chi$ is shown in figure 5. The index $n$ for all the frequencies has values ranging from 0.77 to 2.40 and the most probable value lies between 1.3 and 1.5 (the average value for the entire period is found

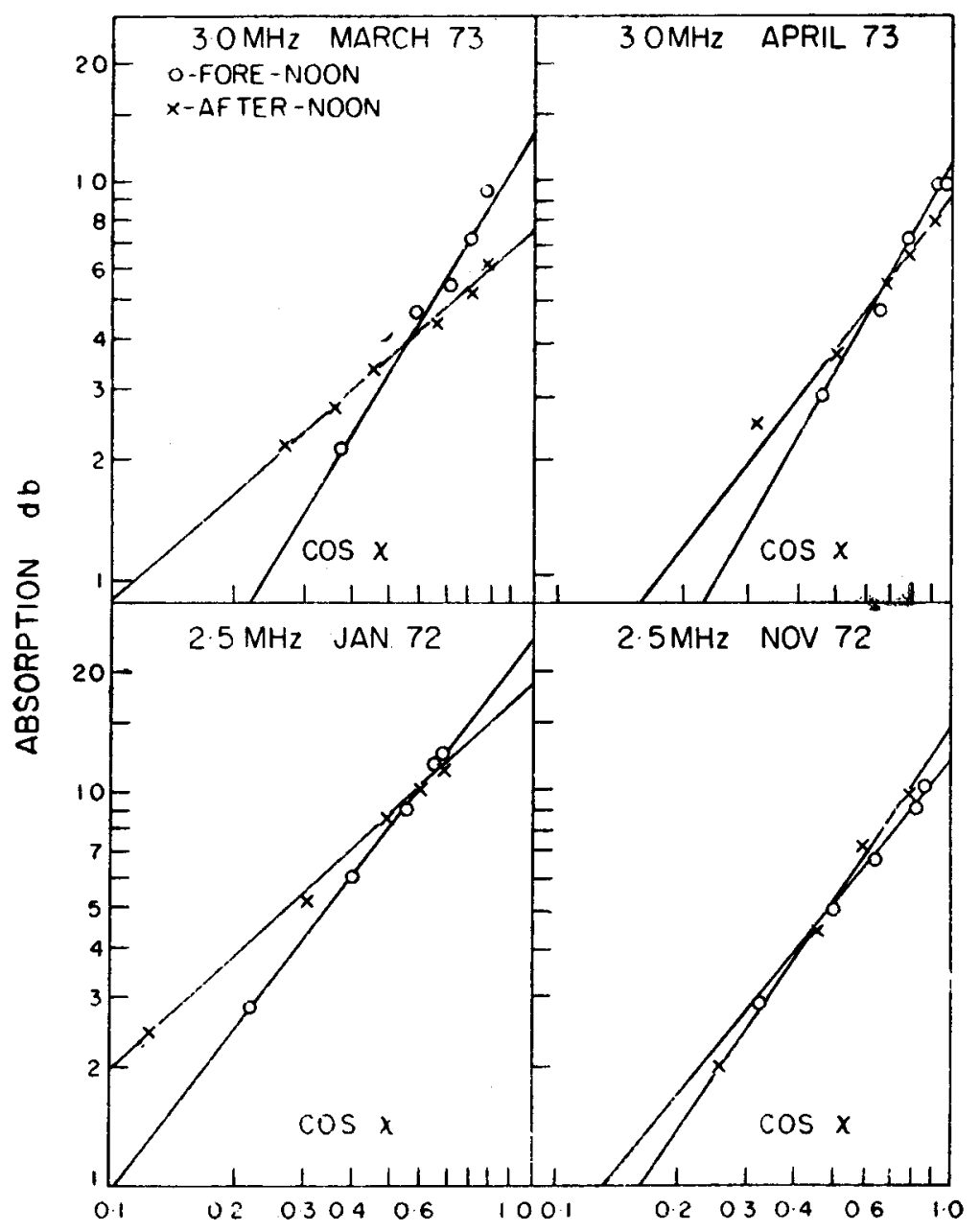

Figure 1. Variation of $D$ region absorption with $\cos x$ in different months. 


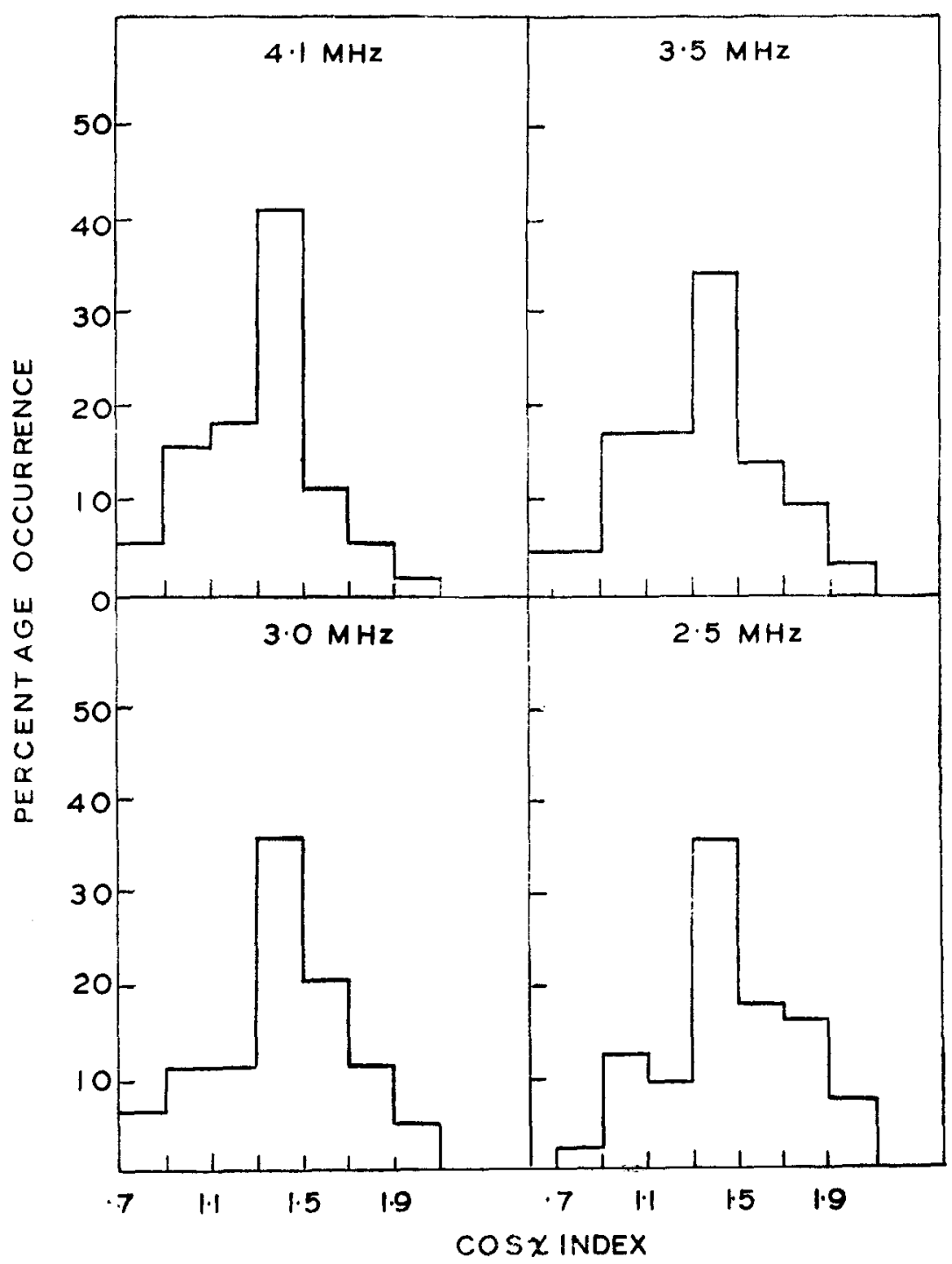

Figure 2. Histograms of $\cos x$ index for $D$ region absorption for different frequencies. 


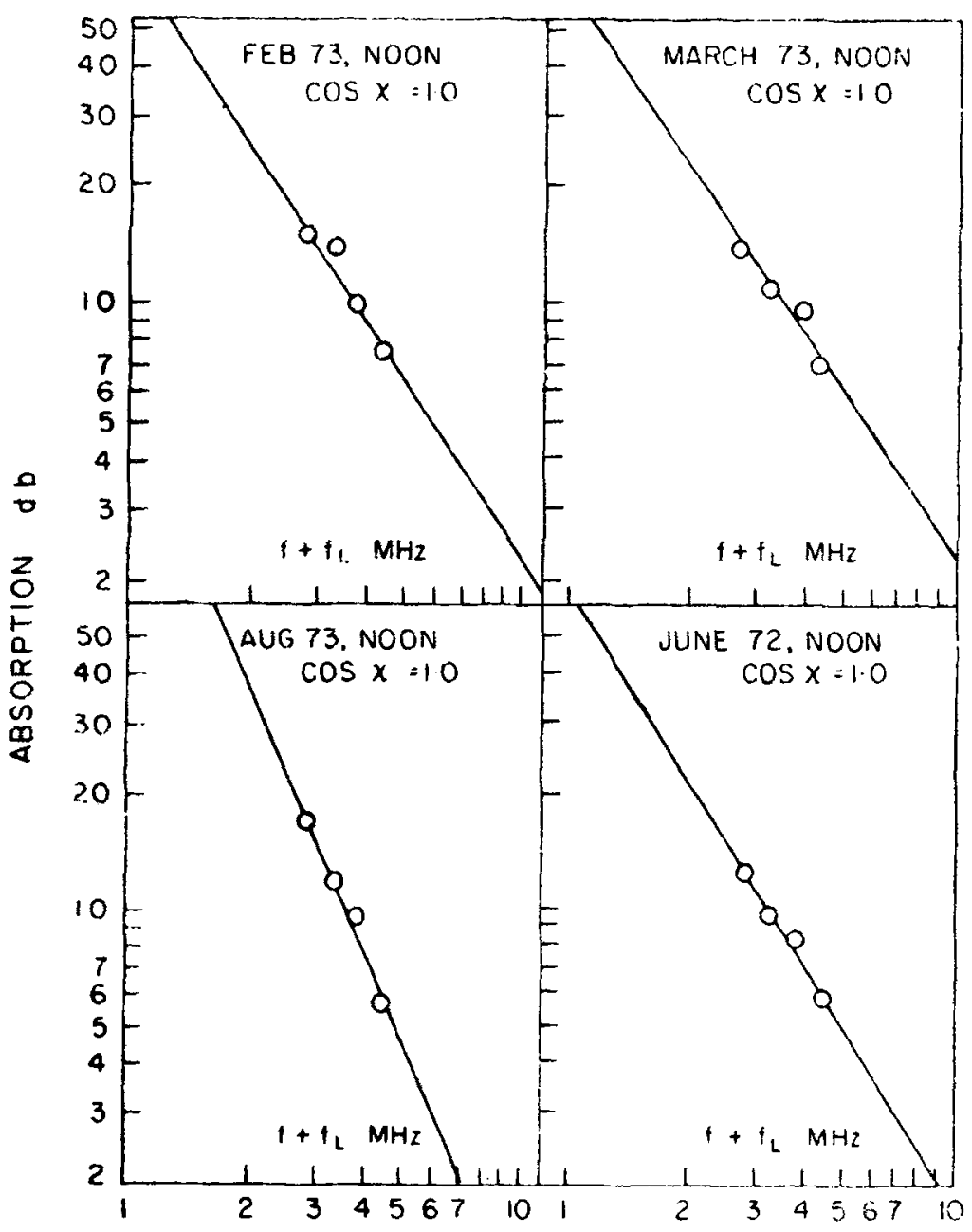

Figure 3. Variation of noon-time $D$ region absorption with effective frequency. 

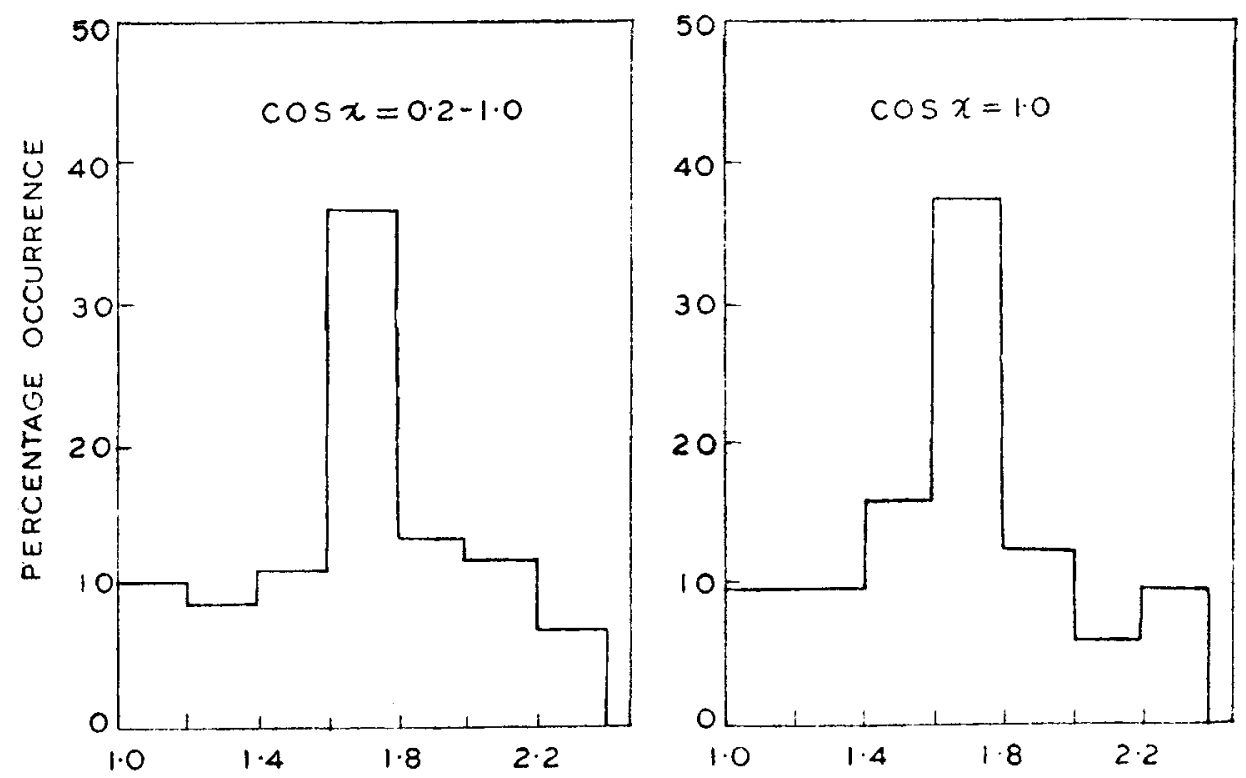

FREOUENCY INDEX, $m$

Figure 4. Histograms of frequency index for $D$ region absorption for $\cos x=1 \cdot 0$ and $\cos x=0.2$ to 1.0 .

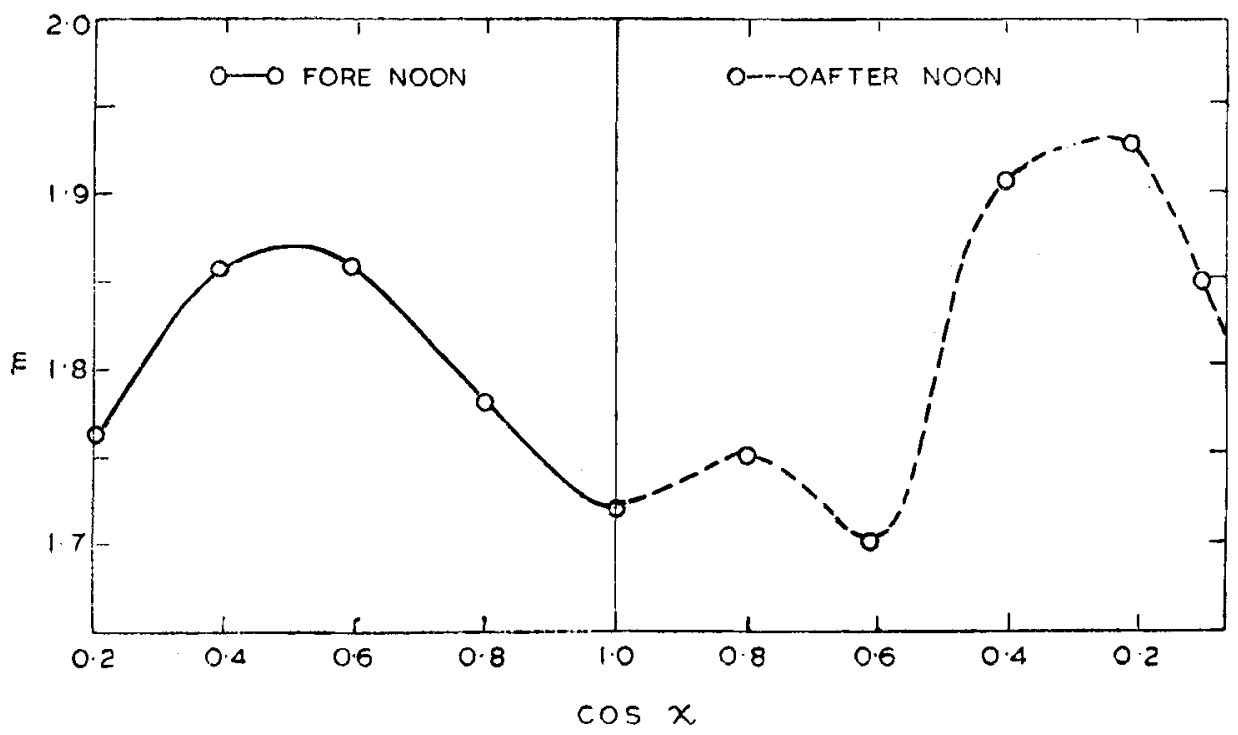

Pigure 5. Variation of frequency index with $\cos x$. 


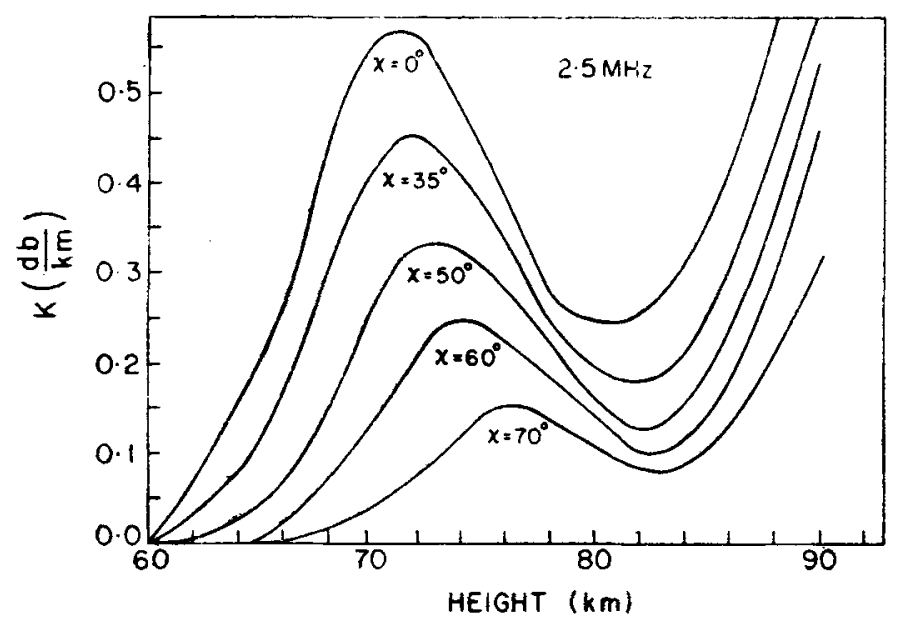

Figure 6. Variation of absorption coefficient with height.

to be $1 \cdot 4$ ). The values of $m$ are distributed in the range 0.97 to $2 \cdot 3$, the mean being 1.76. The most probable value is found to be within 1.6 and $1 \cdot 8$. The present values of $n$ and $m$ agree with the earlier reported values for middle latitude stations.

Computations have been made for absorption variations using the $N(h)$ profiles given by Aikin et al (1972), a model $v(h)$ profile as given in CIRA (1965) and the magnato-ionic theory of Sen-Wyller (1960). The calculated variation of absorption coefficient for $2.5 \mathrm{MHz}$ at different $\chi$ values is shown in figure 6 . The index $m$ which depends weakly on $\chi$ has a variation from $1 \cdot 71$ at $\chi=0$ to 1.87 at $\chi=$ $70^{\circ}$.

The calculated and experimental values of $n$ and $m$ agree.

\section{References}

Aikin A C, Goldberg G A, Somayajulu Y V and Avadhanulu M B 1972 J. Atmos. Terr. Phys. 34 1483

CIRA 1965 COSPAR International Reference Atmosphere (Amsterdam: NH Publishing Co.)

Jaeger J C 1947 Proc. Phys. Soc. (London) 5987

Jain S L 1973 Ph.D. Thesis, University of Udaipur

Jain S L and Rai R K 1974 J. Inst. Engrs. (India) 55 ETI 23

Jain S L and Rai R K 1976 J. Inst. Engrs. (India) 57 ETJ 22

Sen H K and Wyller A A 1960 J. Geophys. Res. 653931 